\title{
Das Gesundheitskapital
}

\author{
Thomas Schramme
}

Online publiziert: 2 . November 2020

(C) Springer-Verlag GmbH Deutschland, ein Teil von Springer Nature 2020

Interessanter als die Frage, warum Jens Spahn seinen Vorschlag, Immunitätspässe einzuführen, wieder zurückgezogen hatte und nun ethisch überprüfen ließ, ist die Frage, warum er den Vorschlag überhaupt gemacht hat. Als Gesundheitsminister war ihm sicherlich bekannt, dass die Idee weder praktikabel noch sinnvoll ist. Welchem Zweck sollte das Ganze dienen?

Spahn hat einen Versuchsballon steigen lassen: Wieviel Widerstand zeigt sich? Wie vehement fällt er aus? Oder finden die Bürger gar Gefallen an der Idee? Bedient man sich der derzeit vorherrschenden medizinischen Begrifflichkeit, so kann man Spahns Versuchsballon als Symptom einer veränderten gesellschaftlichen Realität begreifen. Unter anderen Rahmenbedingungen wäre die Idee niemals losgelassen worden. Doch wie konnten wir in eine gesellschaftliche Situation geraten, in der ein solches Ansinnen ernsthaft von einem führenden Politiker vorgebracht werden kann?

Ein Anhaltspunkt ergibt sich aus einem interessanten Detail der ganzen Affäre. Dieses besteht in der auf den ersten Blick durchaus sinnvollen Anfrage Spahns an den Deutschen Ethikrat, sich der moralischen Bewertung der Pässe anzunehmen. Sind die Bescheinigungen nicht doch vielleicht eine gute Sache? Man wird doch mal drüber diskutieren dürfen (Deutscher Ethikrat 2020).

Doch der Ethikrat ist kein Ethikseminar. Worüber hier diskutiert wird, hat nicht nur rein akademischen Wert, so dass man frei von der Leber weg herumphantasieren könnte. Was hier diskutiert wird schafft Realitäten; eine neue Normalität. Wo wir sinnvoll über die ethische Bewertung von Immunitätspässen debattieren können, da haben wir gesellschaftlich bereits einen wesentlichen Schritt vollzogen: Wir haben

Prof. Dr. T. Schramme ( $₫)$

Department of Philosophy, University of Liverpool, Mulberry Court - Mulberry

Street, Liverpool, L69 7ZY, Großbritannien

E-Mail: t.schramme@liverpool.ac.uk 
individuelle Gesundheit in einen sozialen Status verwandelt. Von einer solchen Warte aus betrachtet ist es durchaus sinnvoll, Gesundheit amtlich zertifizieren zu lassen.

Wozu diese Entwicklung führt, kann man bereits heute erleben, auch ohne amtliches Zeugnis. Wer gesund ist, kann Dinge tun, die Kranke nicht können. Gesundheit ist ein Gut - es zu besitzen stellt ein anzustrebendes Ziel dar. Soweit ist daran nichts Besonderes, das war schon immer so und stellt einen der wesentlichen Gründe dar, warum solidarisch finanzierte Institutionen der Gesundheitsversorgung eingeführt wurden. Krankheit kann jeden treffen, darin sind alle Bürger gleich. Individuelle Gesundheit sollte wiederhergestellt werden. Das gebietet die Gleichheit aller Bürger.

Doch Gesundheit ist auch ein komparatives Gut - ein Vorteil. Gesunden Menschen geht es nicht nur besser als Kranken im Sinne des Wohlbefindens; ihnen fällt es auch leichter, um knappe Güter zu konkurrieren. Diese Tatsache kann man an einem einfachen Beispiel illustrieren: Ein kranker Marathonläufer wird eine geringere Wahrscheinlichkeit als sein gesunder Konkurrent haben, das Rennen zu gewinnen. Der individuelle Gesundheitsstatus wird in dieser Perspektive zu einer Art Ausstattung eines Menschen; Gesundheit wird persönliches Kapital.

Heutzutage sind sehr viele gesellschaftlich gesteuerte Güter relevant für ökonomischen Erfolg: schulische und universitäre Ausbildung, gesellschaftliche Herkunft, Aussehen, sozio-ökonomischer Status und natürlich Gesundheit. Wo Gesundheit einen Wettbewerbsvorteil darstellt, wie in unserer modernen Gesellschaft, dort vergleicht man seinen Gesundheitsstaus mit dem der anderen Bürger. Es ist nicht mehr nur relevant für mein Leben, wie gesund ich bin, sondern auch, wie gesund andere sind. Wer gesünder als ich ist, kann wahrscheinlich eine bessere Ausbildung erreichen, einen anstrengenderen Job ausüben oder sich besser konzentrieren und dadurch mehr leisten.

Somit sind wir in einer Situation gelandet, in der Gesundheit nicht einfach mit Krankheit kontrastiert wird. Der individuelle Gesundheitszustand wird auf einer imaginären Skala dem der anderen zum Vergleich gestellt. Man kann nun gesünder sein als andere, also einen Vergleich ziehen zwischen individuellen Gesundheitszuständen.

Wenn zwei Menschen keine Krankheit haben, dann sind sie beide gesund so dachte man lange Zeit. Jetzt ist ebenfalls die Wahrscheinlichkeit zu erkranken relevant, die Fitness oder Resilienz. Wer eine bessere Gesundheitsdisposition als ein anderer hat, ist gesünder, auch wenn beide keine Krankheit haben. In einem Konkurrenzsystem kommt hinzu: Wer die beste Gesundheit hat, gewinnt.

Immunität gegenüber einem Virus ist ein Wettbewerbsvorteil. Darin ähnelt die derzeitig diskutierte Eigenschaft jeder anderen vergleichsweise besseren Gesundheitsdisposition. Die Zertifizierung der Immunität, wenn sie denn möglich wäre, führt nicht zu größerer Ungleichheit. Vielmehr basiert die ganze Idee bereits auf der Diagnose einer Ungleichheit; einer Ungleichheit, die weit über den Unterschied zwischen krank und gesund hinausreicht.

Medizinhistoriker haben diese Entwicklung mit dem Beispiel des Gelbfiebers verglichen, das in den USA lange Zeit verbreitetet war (Olivarius 2020). Dort hatten nur ,akklimatisierte“ Bürger, also solche, die immun gegen Ansteckung waren, 
Zugang zum Arbeitsmarkt. In diesem Sinne existierte tatsächlich bereits damals ein „Immunkapital“ - und genau dieses Gut debattieren wir auch heute.

Hat man diesen Blickwinkel einmal eingenommen, dann ergeben sich weitere sinnvolle Erklärungen von vermeintlich seltsamen Entwicklungen der jüngeren Zeit. Nehmen wir zunächst das social distancing und Verstöße dagegen. Wer die Regeln nicht einhält, gefährdet nicht einfach die Gesundheit anderer; er könnte ihr Leben gefährden. Insofern scheinen selbst vehemente Reaktionen verständlich.

Doch was ist mit weniger drastischen Gefährdungen anderer? Sich mit einer normalen Erkältung in einen beengten Raum zu begeben, gefährdet ebenfalls die Gesundheit anderer. Es droht ein Schaden; eine Verringerung des individuellen Gesundheitskapitals, also ein Wettbewerbsnachteil durch das Verschulden einer anderen Person. So gesehen, sind Sanktionen ebenfalls angezeigt. Der Wettbewerb um das beste Gesundheitskapital rechtfertigt, für die Einhaltung der Regelverstöße zu sorgen.

Hinzu kommen indirekte Schädigungen. Wo jemand selbstverschuldet gesundheitliche Ressourcen verbraucht, stehen diese den Mitbürgern nicht mehr für ihre Gesundheitsverbesserung zur Verfügung. Deren individuelles Gesundheitskapital wird indirekt verringert. Kein Wunder, dass Ökonomen hier von einem moral hazard sprechen, also einer Art moralischen Gefährdung.

Derzeit schlägt die Stunde der Epidemiologen. Sie untersuchen die Verbreitung von Krankheit in der Bevölkerung. Sozialepidemiologen haben zudem aufgezeigt, welchen gravierenden Einfluss sozio-ökonomische Faktoren auf den Gesundheitsstatus von Menschen haben. Stress, mangelnde Ausbildung, beengte Wohnsituationen - all dies sind Beispiele für soziale Determinanten der Gesundheit. Völlig zurecht wird nun darauf hingewiesen, dass aufgrund dieser gesellschaftlichen Faktoren ein wichtiges Gut, nämlich individuelle Gesundheit, ungleich verteilt ist. Einfach gesagt: Ökonomische Ungleichheit führt zu ungleichem Gesundheitskapital.

Da viele Bürger abgeneigt sind, einen nachteiligen Gesundheitsstatus einfach hinzunehmen, ergeben sich aus dieser Betrachtung alternative Skandalisierungen bereits akzeptierter Ungleichheiten. Es erscheint nicht mehr hinnehmbar, dass einige Menschen ungebildet sind oder in ärmlichen Quartieren hausen, wenn dies mit einem schlechteren Gesundheitsstatus einhergeht. Dieser an sich politisch wohlgemeinte Schachzug - die Ablehnung ökonomischer Gefälle aufgrund ihrer gesundheitlichen Auswirkungen - beruht allerdings leider ebenso auf der neuen Logik. Individuelle Gesundheit wird zu einem persönlichen Kapital - auch wenn das politische Augenmerk auf den Benachteiligten liegt.

Hat man diesen Schritt einmal vollzogen, dann ist die Bedeutung der Gesundheit vom Leben des betroffenen Menschen entkoppelt. Gesundheit hat sich in eine Relation zwischen Konkurrenten verwandelt. Welche politischen Vorlieben man auch pflegt, ob man gegen Ungleichheit agitiert oder nicht, ändert nichts an dem entscheidenden Befund: Ein Aspekt des menschlichen Lebens wurde ökonomisiert.

Interessenkonflikt T. Schramme gibt an, dass kein Interessenkonflikt besteht. 


\section{Literatur}

Deutscher Ethikrat (2020) Stellungnahme: Immunitätsbescheinigungen in der Covid-19-Pandemie. https:// www.ethikrat.org/fileadmin/Publikationen/Stellungnahmen/deutsch/stellungnahme-immunitaetsbe scheinigungen.pdf. Zugegriffen: 13. Okt. 2020

Olivarius K (2020) The dangerous history of immunoprivilege. New York Times 12.04.2020. https://www. nytimes.com/2020/04/12/opinion/coronavirus-immunity-passports.html. Zugegriffen: 12. Okt. 2020 